\title{
Structure and thermal expansion of liquid bismuth
}

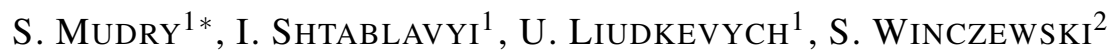 \\ ${ }^{1}$ Physics of Metals Department, Ivan Franko National University of Lviv, 8 Kyrylo and Mephodiy St., 79005 Lviv, Ukraine \\ ${ }^{2}$ Department of Solid State Physics, Faculty of Applied Physics and Mathematics, Gdansk University of Technology, 11/12 \\ Narutowicza St., 80-233 Gdansk, Poland
}

\begin{abstract}
Experimental structural data for liquid Bi were used for estimation of the main structure parameters as well as the thermal expansion coefficient both in supercooled and superheated temperature ranges. It was shown that the equilibrium melt had a positive thermal expansion coefficient within a temperature range upon melting and a negative one at higher temperatures. The former was related to structure changes upon melting, whereas the latter with topologic disordering upon further heating. It was found that the superheated melt had a negative thermal expansion coefficient. The results obtained from structural data were compared with the thermal expansion coefficient calculated from the data of density for liquid Bi.
\end{abstract}

Keywords: liquid metal; short range order; thermal expansion; structure transformation

(C) Wroclaw University of Technology.

\section{Introduction}

Thermophysical properties of matter are important both from the fundamental and practical application point of view for physics, chemistry and materials science. It is known that materials generally expand upon heating. Thermal expansion is a measure of dilation and describes the behavior of various materials with changing temperature, being closely related to the structure, chemical interaction of neighboring atoms, the resulting mechanical properties, the transport coefficient, etc. In this regard, experimental results on the thermal expansion coefficient yield valuable information which is necessary for application of the materials in various areas.

Traditionally, most attention in the study of thermal expansion has been paid to solid materials with a crystalline structure in which a periodic atomic arrangement allowed developing a theory and using it for the interpretation of experimental data [1]. Contrary to crystalline materials, amorphous solids and liquids have a short range order only and for this reason the theoretical background for their thermal expansion is significantly less

*E-mail: sihor@ukr.net developed. New areas of application of such disordered materials need deeper knowledge of their properties including the thermal expansion behavior, considering it not only as a macroscopic characteristics, but also as depending on the distribution of neighboring atoms. It is of fundamental importance to establish the relation between the thermal expansion properties of a melt and the changes of atomic arrangement with respect to the nearest neighbors.

Most data on the thermal expansion of liquid metals and alloys have been obtained by means of density and dilatometer measurements, whereas there are only few works, in which this parameter is studied by diffraction methods at the atomic level [2-4]. However, these works are occasional so there is a necessity for a detailed study of the phenomenon of thermal expansion of liquid metals and alloys. Particularly noteworthy are the metals with loose packing of atoms in the crystalline state, whose atomic structure after melting may become of close packing. One of such metals is bismuth.s

An interest in the study of the structure of liquid bismuth has recently increased significantly owing to the investigations of the phenomenon of structural transformations of metals and alloys in the liquid state [5-10]. 
In the solid state, $\mathrm{Bi}$ has a loose packing (a rhombohedral structure with three-fold coordination symmetry). The structure consists of two interpenetrating face-centered sub-lattices, one of which is slightly displaced from the mid point position of the other [11]. Upon melting there is a temperature range, within which atomic arrangement tends to be more close packed than in the solid state, which causes an anomalous change of the structure parameters. This transformation of the structure is confirmed by a slight increase in the density of liquid bismuth at temperatures close to the melting point.

The structure of liquid Bi has been well studied and most researchers $[12,13]$ pointed out that this element has the structure different than close packed metals. The main difference is in the existence of a shoulder on the right hand side of the structure factor (SF) and a smaller number of neighbors, which according to the results of different authors lies within the range of 7.5 to 9.0 at a temperature close to the melting point. There are few models which represent the structure of liquid $\mathrm{Bi}$ as well as other semimetals, including the double structure $[14,15]$, the distorted structure [1618] and the quasicrystalline structure [19]. It is unclear which of them better describes the real structure of liquid $\mathrm{Bi}$ and explains the thermal expansion behavior.

In the literature [20, 21], the structure change of $\mathrm{Bi}$ during the liquid - supercooled liquid - solid transition process at the temperature range of 510 to $520 \mathrm{~K}$ was studied by means of the energy scanning $\mathrm{X}$-ray method. It has been suggested that there exists a pre-crystalline state with a rhombohedral structure which is not the same as the one just before the transition to the crystalline phase. The results of structure studies are consistent with the physical properties measurements and first of all with the temperature dependence of density which reveals an increase of $3.4 \%$ upon melting. In the literature [22] it has been indicated that there are no structural changes that would suggest crystallization in the higher temperature range (528 to $548 \mathrm{~K}$ ). Due to this, there is a reason for a detailed study of the liquid bismuth structure in a wide temperature range.

This work deals with an estimation of the thermal properties of liquid bismuth using the structure data obtained by the X-ray diffraction method. Since the supercooled liquid exists in a metastable state, we also estimated the thermal expansion coefficient and compared it with the one for the temperature range, where an equilibrium liquid exists.

\section{Fundamental}

The volume thermal expansion coefficient can be described as:

$$
\beta=\frac{1}{V} \frac{d V}{d T}=-\frac{1}{\rho} \frac{d \rho}{d T}
$$

However, the coefficient of thermal expansion of disordered systems calculated according to this formula gives only its average value. Therefore, for a more detailed study of the thermal expansion processes it is necessary to study the temperature changes of parameters at the atomic level.

By means of X-ray diffractometry, the coefficient of thermal expansion of crystalline materials is determined by the change in lattice parameters with temperature. For disordered systems, which are characterized by short-range atomic ordering, the lattice parameter concept looses its meaning. Therefore, we have to use statistical methods to calculate the interatomic distances in respect of disordered systems. For this purpose, the so-called Pair Correlation Function (PCF) is used. Using this function, we can determine the most probable interatomic distances $r_{1}$ as a position of the PCF first maximum, mean interatomic distances $\left\langle r_{1}\right\rangle$ as well as root mean square interatomic distances $\sqrt{\left\langle\mathrm{r}_{1}^{2}\right\rangle}$ which can be calculated on the basis of the PCF first peak profile and the first coordination shell radius $r_{\min }$ as a position of the PCF first minimum. The last three parameters, as we suppose, are more sensitive to structure changes upon melting of $\mathrm{Bi}$ and similar elements, in comparison with the most probable interatomic distance, are commonly used for the estimation of thermal expansion in liquid metals [4]. 
Mean interatomic distances $\left\langle\mathrm{r}_{1}\right\rangle$ and root mean square interatomic distances $\sqrt{\left\langle\mathrm{r}_{1}^{2}\right\rangle}$ can be calculated by the formulas:

$$
\begin{gathered}
\left\langle r_{1}\right\rangle=\frac{\int_{r_{0}}^{r_{\min }} r g(r) d r}{\int_{r_{0}}^{r_{\min }} g(r) d r} \\
\sqrt{\left\langle r_{1}^{2}\right\rangle}=\sqrt{\frac{\int_{\int_{r_{0}}^{r_{\min }} r^{2} g(r) d r}^{r_{\min }} g(r) d r}{r_{0}}}
\end{gathered}
$$

where $r_{\min }$ is the first minimum with respect to the principal peak of PCF. As has been mentioned above, this parameter is assumed to be equal to the radius of the first coordination shell in the liquid state.

The thermal expansion coefficient can be defined also using the temperature dependence of the coordination numbers [2] and the atomic volume.

The atomic volume in the first coordination shell has been calculated as $\mathrm{V}_{\mathrm{a}}=\frac{4}{3 \mathrm{Z}} \pi \mathrm{r}_{\min }^{3}$, where $\mathrm{Z}$ is a coordination number $\left(\mathrm{Z}=\int_{\mathrm{r}_{1}}^{\mathrm{r}_{2}} 4 \pi r^{2}\langle\rho\rangle \mathrm{g}(\mathrm{r}) \mathrm{dr}\right)$. Depending on the limits of integration, in this work we present two values of the coordination numbers $\mathrm{Z}$ and $\mathrm{N}$.

Using these parameters, the thermal expansion coefficient can be calculated according to the relations (we propose appropriate designations for the coefficients calculated using various parameters):

$$
\begin{gathered}
\beta_{\rho}=-\frac{1}{\rho} \frac{d \rho}{d T} \\
\beta_{V}=\frac{1}{V} \frac{d V}{d T} \\
\beta_{m p}=\frac{3}{r_{1}} \frac{d r_{1}}{d T} \\
\beta_{m d}=\frac{3}{\left\langle r_{1}\right\rangle} \frac{d\left\langle r_{1}\right\rangle}{d T}
\end{gathered}
$$

$$
\begin{gathered}
\beta_{r m s}=\frac{3}{\left\langle r_{1}^{2}\right\rangle^{\frac{1}{2}}} \frac{d\left(\left\langle r_{1}^{2}\right\rangle\right)^{\frac{1}{2}}}{d T} \\
\beta_{f c s}=\frac{3}{r_{\min }} \frac{d r_{\min }}{d T} \\
\beta_{V a}=\frac{1}{V_{a}} \frac{d V_{a}}{d T} \\
\beta_{N}=\frac{1}{N} \frac{d N}{d T} \\
\beta_{Z}=\frac{1}{Z} \frac{d Z}{d T}
\end{gathered}
$$

\section{Experimental}

The thermal expansion coefficient was estimated from the data on the temperature dependence of mean interatomic distances $\left\langle\mathrm{r}_{1}\right\rangle,\left\langle\mathrm{r}_{1}^{2}\right\rangle$, the atomic volume $\mathrm{V}_{\mathrm{a}}$ and the coordination number $\mathrm{Z}$, which were determined from pair correlation functions (PCF). For comparison, we calculated the coefficient of thermal expansion using the data on density [23].

The XRD measurements were carried out by means of a high-temperature X-ray diffractometer. $\mathrm{CuK} \alpha$ radiation monochromatized with an $\mathrm{LiF}$ single crystal and Bragg-Brentano focusing geometry in the $2 \theta$ range from $10^{\circ}$ up to $120^{\circ}$ were used. Intensity curves were corrected for polarization and incoherent scattering [24]. After this procedure they were normalized to electron units by the Krogh-Moe method [25]. A detailed description of the experimental technique was reported [26]. The scattered intensity was measured with an accuracy higher than $2 \%$. The main structure parameters obtained from the structure factor (SF) and the pair correlation function were calculated according to Mayo et al. [27]. The typical uncertainty in PCF obtained by this method is of the order of a few percent. The main structure parameters obtained from them were analyzed.

For the X-ray diffraction investigation we used purified samples of $\mathrm{Bi}(99.999)$. 


\section{Results and discussion}

The PCFs obtained for liquid Bi (Fig. 1) are quite different from the ones in wide temperature intervals ( 573 to $1223 \mathrm{~K}$ ) and ( 522 to $512 \mathrm{~K}$ ). The former corresponds to the equilibrium liquid and the latter - to the supercooled (metastable) one. As it can be seen in this figure, a decrease in the principal peak height and an increase in the width are observed with increasing temperature. The most probable interatomic distance $r_{1}$ is less sensitive to temperature. The distance rather slightly decreases with heating, contrary to the commonly observed increasing of this parameter for most crystalline solids. Therefore, the temperature disordering of the liquid structure is not consistent with a typical behavior of thermal expansion properties for crystals.

Other features are observed for supercooled liquid bismuth (Fig. 1b) that was studied in detail in the literature [20] by means of energy scanning Xray method. The principal peak has two shoulders, notably transformed upon decreasing temperature. Namely, the first shoulder increases, whereas the other decreases. These shoulders can appear as a result of pre-crystallization processes in supercooled bismuth. As it can be seen in Fig. 1 their position well coincides with the position of coordination spheres in the crystalline bismuth. The principal peak slightly decreases its height and shifts to larger distances upon cooling. Therefore, similar behavior as for the equilibrium liquid is generally observed in case of the supercooled state.

In order to carry out a more detailed study of the effect of temperature on the atomic arrangement of liquid $\mathrm{Bi}$ we determined a thermal expansion coefficient using the temperature dependencies of different structure parameters (most probable interatomic distances $r_{1}$, mean interatomic distances $\left\langle\mathrm{r}_{1}\right\rangle$, root mean square interatomic distances $\sqrt{\left\langle\mathrm{r}_{1}^{2}\right\rangle}$, the radius of the first coordination shell, the atomic volume in the first coordination shell), calculated from PCFs.

As it is seen in Fig. 2, the temperature dependence of $r_{1}$ shows a slight decrease over a wide temperature region. Similar dependence is

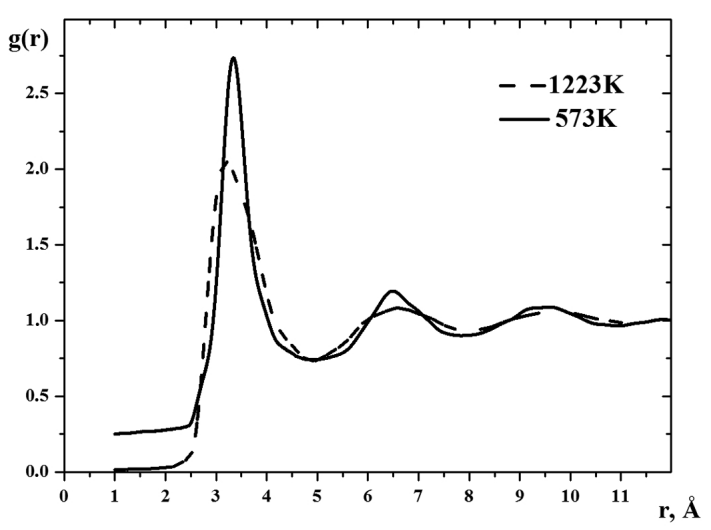

(a)

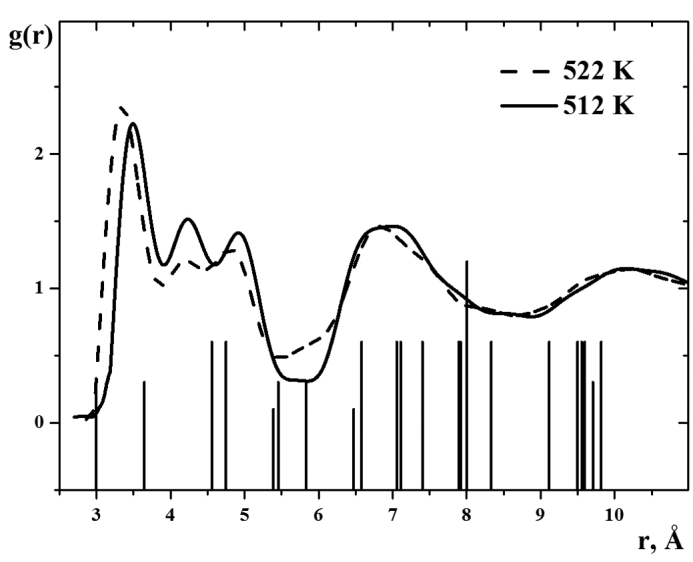

(b)

Fig. 1. Effect of overheating (a) and supercooling (b) of liquid $\mathrm{Bi}$ on its atomic distribution.

observed for the supercooled state of the melt. Generally, cooling of molten Bi from high temperatures to those corresponding to supercooled liquid reveals no notable changes of this parameter.

Different behaviors take place in the temperature dependences of the mean interatomic distance and the root mean square interatomic distance. For an equilibrium liquid $\left\langle\mathrm{r}_{1}\right\rangle$ and $\sqrt{\left\langle\mathrm{r}_{1}^{2}\right\rangle}$ show small increases within some temperature range close to the crystallization point. At higher temperatures a slight decrease in these parameters as well as in the radius of the first coordination sphere is observed. Thus, during melting of $\mathrm{Bi}$, the structure transformation occurs at heating up to some temperature point. Upon reaching this point the mean interatomic distance and radius of first coordination shell decrease that is an evidence of different 


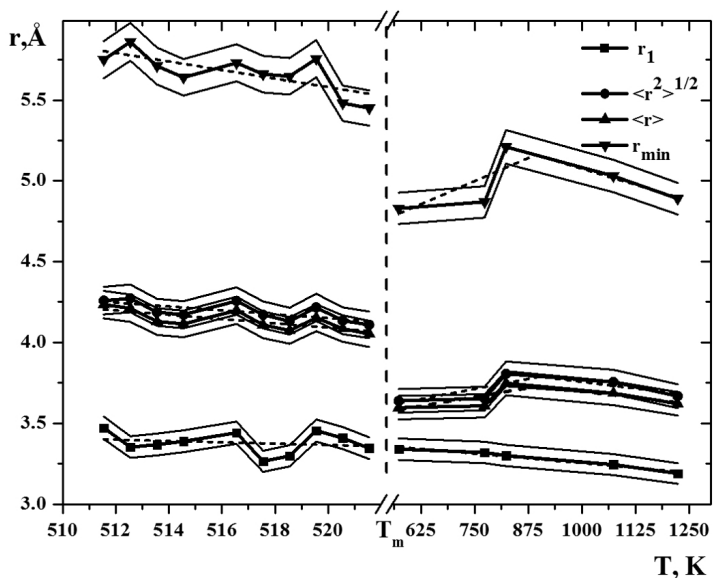

(a)

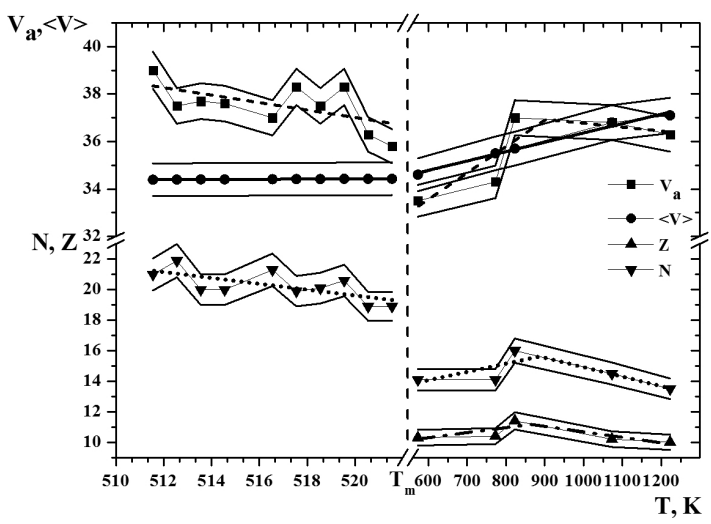

(b)

Fig. 2. Temperature dependence of main structure parameters for liquid $\mathrm{Bi}$.

thermal behavior of the molten metal structure. A similar dependence is observed for the coordination numbers and the atomic volume (Fig. 2b).

Contrary to the equilibrium liquid, a supercooled melt reveals no subranges in the temperature dependence of mean distances and radius of the first coordination sphere. There is a gradual increase in these parameters with cooling.

The obtained results allowed us to consider distances $\left\langle\mathrm{r}_{1}\right\rangle$ and mean square interatomic distances $\sqrt{\left\langle r_{1}^{2}\right\rangle}$ as highly informative characteristics of structure changes upon melting of $\mathrm{Bi}$ and similar elements in comparison with the most probable interatomic distance. It follows more evidently from the analysis of the volume thermal expansion coefficient (Fig. 3).

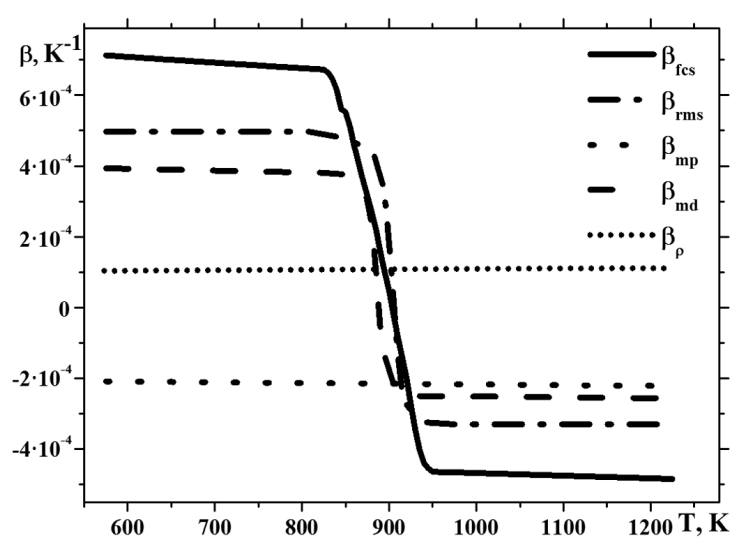

(a)

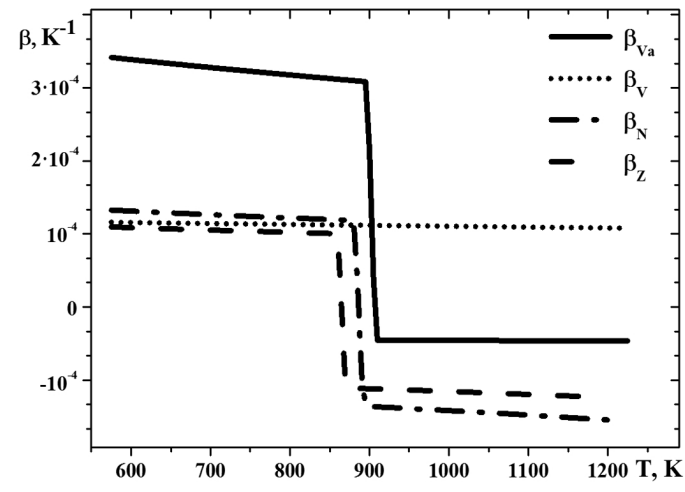

(b)

Fig. 3. Temperature dependence of thermal expansion coefficient for liquid $\mathrm{Bi}$.

As seen in Fig. 3 and Table 1, the coefficient of thermal expansion calculated from the temperature dependence of the most probable interatomic distances $\beta_{\mathrm{mp}}$, is negative. Our results are in agreement with the data obtained in the literature [4], where the authors pointed out that liquid metals have a negative thermal expansion coefficient over a wide temperature range and the thermal expansion of liquids can be explained by an increase in the free volume on the boundary between the first and second coordination shells.

Moreover, according to our calculations, the coefficient of thermal expansion obtained on the basis of temperature dependences of mean interatomic distances becomes negative at temperatures of several hundred degrees higher than the melting point of $\mathrm{Bi}$. The temperature interval of the positive 
Table 1. Numerical values of thermal expansion coefficient for liquid Bi.

\begin{tabular}{|c|c|c|c|c|c|c|c|c|c|}
\hline $\begin{array}{c}\mathrm{T} \\
{[\mathrm{K}]}\end{array}$ & $\begin{array}{c}\beta_{\mathrm{fcs}} \times 10^{4} \\
{\left[\mathrm{~K}^{-1}\right]}\end{array}$ & $\begin{array}{c}\beta_{\mathrm{rms}} \times 10^{4} \\
{\left[\mathrm{~K}^{-1}\right]}\end{array}$ & $\begin{array}{c}\beta_{\mathrm{mp}} \times 10^{4} \\
{\left[\mathrm{~K}^{-1}\right]}\end{array}$ & $\begin{array}{c}\beta_{\mathrm{md}} \times 10^{4} \\
{\left[\mathrm{~K}^{-1}\right]}\end{array}$ & $\begin{array}{c}\beta_{\rho} \times 10^{4} \\
{\left[\mathrm{~K}^{-1}\right]}\end{array}$ & $\begin{array}{c}\beta_{\mathrm{Va}} \times 10^{4} \\
{\left[\mathrm{~K}^{-1}\right]}\end{array}$ & $\begin{array}{c}\beta_{\mathrm{V}} \times 10^{4} \\
{\left[\mathrm{~K}^{-1}\right]}\end{array}$ & $\begin{array}{c}\beta_{\mathrm{N}} \times 10^{4} \\
{\left[\mathrm{~K}^{-1}\right]}\end{array}$ & $\begin{array}{c}\beta_{\mathrm{Z}} \times 10^{4} \\
{\left[\mathrm{~K}^{-1}\right]}\end{array}$ \\
\hline 575 & 7.13 & 4.97 & -2.09 & 3.93 & 1.04 & 3.35 & 1.14 & 1.30 & 1.07 \\
\hline 600 & 7.08 & 4.97 & -2.09 & 3.92 & 1.04 & 3.32 & 1.13 & 1.29 & 1.06 \\
\hline 625 & 7.04 & 4.97 & -2.10 & 3.91 & 1.04 & 3.29 & 1.13 & 1.27 & 1.06 \\
\hline 650 & 7.00 & 4.97 & -2.10 & 3.89 & 1.05 & 3.27 & 1.13 & 1.26 & 1.05 \\
\hline 675 & 6.96 & 4.97 & -2.11 & 3.88 & 1.05 & 3.24 & 1.12 & 1.25 & 1.04 \\
\hline 700 & 6.92 & 4.97 & -2.11 & 3.87 & 1.05 & 3.21 & 1.12 & 1.24 & 1.03 \\
\hline 725 & 6.88 & 4.97 & -2.12 & 3.86 & 1.06 & 3.19 & 1.12 & 1.23 & 1.02 \\
\hline 750 & 6.84 & 4.97 & -2.13 & 3.84 & 1.06 & 3.16 & 1.11 & 1.22 & 1.02 \\
\hline 775 & 6.80 & 4.97 & -2.13 & 3.83 & 1.06 & 3.14 & 1.11 & 1.21 & 1.01 \\
\hline 800 & 6.76 & 4.97 & -2.14 & 3.82 & 1.07 & 3.11 & 1.11 & 1.19 & 1.00 \\
\hline 825 & 6.73 & 4.94 & -2.14 & 3.81 & 1.07 & 3.09 & 1.10 & 1.18 & 0.99 \\
\hline 835 & 6.45 & 4.86 & -2.14 & 3.80 & 1.07 & 3.08 & 1.10 & 1.18 & 0.99 \\
\hline 845 & 5.60 & 4.79 & -2.14 & 3.77 & 1.07 & 3.07 & 1.10 & 1.18 & 0.98 \\
\hline 855 & 5.12 & 4.72 & -2.15 & 3.70 & 1.07 & 3.06 & 1.10 & 1.17 & 0.99 \\
\hline 865 & 4.07 & 4.61 & -2.15 & 3.52 & 1.07 & 3.05 & 1.10 & 1.17 & -0.05 \\
\hline 875 & 3.16 & 4.46 & -2.15 & 3.06 & 1.08 & 3.04 & 1.10 & 1.16 & -1.09 \\
\hline 885 & 2.20 & 4.21 & -2.15 & 1.02 & 1.08 & 3.03 & 1.10 & 0.33 & -1.09 \\
\hline 895 & 0.98 & 3.11 & -2.15 & -1.38 & 1.08 & 3.02 & 1.10 & -1.33 & -1.09 \\
\hline 905 & -0.20 & 0.25 & -2.16 & -2.14 & 1.08 & 0.38 & 1.09 & -1.34 & -1.10 \\
\hline 915 & -1.42 & -2.42 & -2.16 & -2.38 & 1.08 & -0.44 & 1.09 & -1.34 & -1.10 \\
\hline 925 & -2.75 & -2.90 & -2.16 & -2.47 & 1.08 & -0.44 & 1.09 & -1.35 & -1.10 \\
\hline 935 & -4.04 & -3.15 & -2.16 & -2.50 & 1.08 & -0.44 & 1.09 & -1.35 & -1.11 \\
\hline 945 & -4.55 & -3.26 & -2.16 & -2.51 & 1.08 & -0.45 & 1.09 & -1.36 & -1.11 \\
\hline 950 & -4.64 & -3.30 & -2.17 & -2.51 & 1.09 & -0.45 & 1.09 & -1.36 & -1.11 \\
\hline 975 & -4.66 & -3.30 & -2.17 & -2.51 & 1.09 & -0.45 & 1.09 & -1.38 & -1.12 \\
\hline 1000 & -4.68 & -3.30 & -2.18 & -2.52 & 1.09 & -0.45 & 1.08 & -1.39 & -1.13 \\
\hline 1025 & -4.70 & -3.30 & -2.18 & -2.52 & 1.10 & -0.45 & 1.08 & -1.40 & -1.14 \\
\hline 1050 & -4.72 & -3.30 & -2.19 & -2.53 & 1.10 & -0.45 & 1.08 & -1.42 & -1.15 \\
\hline 1075 & -4.74 & -3.30 & -2.19 & -2.53 & 1.10 & -0.45 & 1.07 & -1.43 & -1.16 \\
\hline 1100 & -4.76 & -3.30 & -2.20 & -2.54 & 1.10 & -0.45 & 1.07 & -1.45 & -1.17 \\
\hline 1125 & -4.77 & -3.30 & -2.20 & -2.55 & 1.10 & -0.45 & 1.07 & -1.47 & -1.18 \\
\hline 1150 & -4.79 & -3.30 & -2.21 & -2.55 & 1.11 & -0.45 & 1.07 & -1.48 & -1.19 \\
\hline 1175 & -4.81 & -3.30 & -2.21 & -2.56 & 1.11 & -0.45 & 1.06 & -1.50 & -1.20 \\
\hline 1200 & -4.83 & -3.30 & -2.22 & -2.56 & 1.11 & -0.45 & 1.06 & -1.52 & -1.22 \\
\hline 1225 & -4.85 & -3.30 & -2.22 & -2.57 & 1.11 & -0.45 & 1.06 & -1.53 & -1.23 \\
\hline
\end{tabular}

thermal expansion coefficient also exists due to the atomic rearrangement upon melting, resulting in the formation of a short range order structure with closely packed atomic distribution.

Contrary to the equilibrium melt, thermal expansion of superheated $\mathrm{Bi}$ does not reveal the region with a positive value of the thermal expansion coefficient. Upon cooling, mean interatomic distances increase due to the formation of metastable clusters with a highly ordered atomic distribution.

It is also seen that the thermal expansion coefficient, estimated from the experimental data on the density shows small positive values which in 
fact are invariable with temperature. This parameter does not show any structure changes with temperature upon melting. Certainly, similar behavior is observed in the temperature dependence of the thermal expansion coefficient determined using the mean atomic volume which is directly related to density. In this case the thermal expansion is influenced by two concurrent factors: increasing the interatomic distances and reducing the free volume during the structure transformation. If we use short range order parameters, the structure changes offer more contribution to the thermal expansion behavior than free volume changes.

\section{Conclusions}

Pair correlation functions at different temperatures were used for the estimation of the thermal expansion coefficient for liquid $\mathrm{Bi}$ both in the equilibrium and supercooled states. It was found that two temperature regions exist for the equilibrium liquid with different signs of this parameter. Upon melting within a narrow temperature range it is positive and becomes negative at higher temperatures. Superheated Bi reveals negative thermal expansion only. The reason for such behavior is the transformation of the short range order upon melting into a more closely packed atomic distribution and increasing the atomic ordering in metastable clusters, created in the superheated state.

\section{Acknowledgements}

S.M., I.S. and S.W. acknowledge the support of the Polish Ministry of Science and Higher Education (Grant IP2012 043972).

\section{References}

[1] In-Kook Suh, Ohta H., Waseda Y., J. Mater. Sci., 23 (1988), 757.

[2] Ocken H., Wagner C.N. I., Phys. Rev., 1 (1966), 122.

[3] BAR'YAKHTAR V. MIKHAILOVA L.E., IL'INSKII A.G., ROMANOVA A.V., KHRISTENKO T.M., JETP, 68 (5) (1989), 811.

[4] Hongbo L., Wang X., CaO Q., Zhang D., Zhang J., Hu T., MaO H.-K., JiAng J.-Z., PNAS, 110 (25) (2013), 10068.
[5] Crichton W.A., Mezouar M., Grande T. Stolen S., GrZECHNIK A., Nature, 414 (2001), 622.

[6] McMillan P.F., Nat. Mater., 1 (2002), 19.

[7] Yargerand J.L., Wolf G.H., Science, 306 (2004), 206.

[8] Wilding M.C., Wilson M., McMillan P.F., Chem. Soc. Rev., 35 (2006), 964.

[9] MCMillan P.F., Wilson M., Wilding M.C., Daisenberger D., Mezouar M., Greaves N.G., J. Phys.-Condens. Mat., 19 (2007), 415101.

[10] Cadien A., Hu Q., Meng Y., Cheng Y., Chen M., Shu J., Mao H., Sheng H., Phys. Rev. Lett., 110 (2013), 125503.

[11] Barrett C.S., Aust. J. Phys., 13 (1960), 209.

[12] Greenberg Y., Yahel E., Caspi E.N., Benmore C., Beuneu B., Dariel M.P., Makov G., EPL 86 (2009), 36004.

[13] Souto J., Alemany M., Gallego L., GonZALez L., Gonzalez D., Phys. Rev. B, 81 (2010), 134201.

[14] Richter H., Breitling G., Adv. Phys., 16 (1968), 293.

[15] ORTON Z.B.R., Z. Naturforsch. A, 34 (1979), 1547.

[16] Krebs H., J. Non-Cryst. Solids, 1 (1969), 455.

[17] Davidovic M., StojiC M., Jovic D.J., J. Phys. CSolid State Phys., 16 (1983), 2053.

[18] Davidovic M., Stojic M., Jovic D.J., J. Non-Cryst. Solids, 61 - 62 (1984), 517.

[19] Bellissent-Funel M.C., Bellisent R., J. NonCryst. Solids, 65 (1984), 383.

[20] Matsuno N., Kamiyama H., Ishil Y., MomiUCHI M., Jpn. J. Appl. Phys., 25 (1986), 275.

[21] Momiuchi M., J. Phys. Soc. Jpn., 55 (1986), 200.

[22] Emuna M., Mayo M., Greenberg Y., CASPi E.N., Beuneu B., Yahel E., Makov G., J. Chem. Phys., 140 (2014), 094502.

[23] Haoran G., Chunjing S., Rui W., Xiaogang Q.I., Ning Z., Chinese Sci. Bull., 15 (2007), 2031.

[24] Cromer D.T., Waber J.T., Acta Crystallogr., 18 (1965), 104

[25] Kroghmoe J., Acta Crystallogr., 9 (1956), 951.

[26] Plevachuk Y., Sklyarchuk V., Yakymovych A., SHTABLAVYI I., Methods and facilities for thermophysical and structure investigations of liquid metallic alloys, in: $6^{\text {th }}$ International Conference Electromagnetic Processing of Materials, Forschungszentrum, DresdenRossendorf-Dresden, 2009, p. 415.

[27] Mayo M., Yahel E., Greenberg Y., Caspi E.N., Beuneu B., Makov G., J. Appl. Crystallogr, 46 (2013), 1582. 